\title{
Time-series lipidomic analysis of the oleaginous green microalga species Ettlia oleoabundans under nutrient stress
}

\author{
E. K. Matich ${ }^{1}$, M. Ghafari ${ }^{2}$, E. Camgoz ${ }^{3}$, E. Caliskann ${ }^{4}$, B. A. Pfeifer ${ }^{5}$, B. Z. Haznedaroglu ${ }^{4}$ \\ and G. E. Atilla-Gokcumen ${ }^{1 *}$ (B)
}

\begin{abstract}
Background: Microalgae are uniquely advantageous organisms cultured and harvested for several value-added biochemicals. A majority of these compounds are lipid-based, such as triacylglycerols (TAGs), which can be used for biofuel production, and their accumulation is most affected under nutrient stress conditions. As such, the balance between cellular homeostasis and lipid metabolism becomes more intricate to achieve efficiency in bioproduct synthesis. Lipidomics studies in microalgae are of great importance as biochemical diversity also plays a major role in lipid regulation among oleaginous species.

Methods: The aim of this study was to analyze time-series changes in lipid families produced by microalga under different nutrient conditions and growth phases to gain comprehensive information at the cellular level. For this purpose, we worked with a highly adaptable, oleaginous, non-model green microalga species, Ettlia oleoabundans (a.k.a. Neochloris oleoabundans). Using a mass spectrometry-based untargeted and targeted metabolomics' approach, we analyzed the changes in major lipid families under both replete and deplete nitrogen and phosphorus conditions at four different time points covering exponential and stationary growth phases.
\end{abstract}

Results: Comprehensive analysis of the lipid metabolism highlighted the accumulation of TAGs, which can be utilized for the production of biodiesel via transesterification, and depletion of chlorophylls and certain structural lipids required for photosynthesis, under nutrient deprived conditions. We also found a correlation between the depletion of digalactosyldiacylglycerols (DGDGs) and sulfoquinovosyldiacylglycerols (SQDGs) under nutrient deprivation.

Conclusions: High accumulation of TAGs under nutrient limitation as well as a depletion of other lipids of interest such as phosphatidylglycerols (PGs), DGDGs, SQDGs, and chlorophylls seem to be interconnected and related to the microalgal photosynthetic efficiency. Overall, our results provided key biochemical information on the lipid regulation and physiology of a non-model green microalga, along with optimization potential for biodiesel and other valueadded product synthesis.

Keywords: Lipidomics, Microalgae, Biochemicals, Metabolomics

\section{Background}

Microalgae species are valuable biomass resources for the advancement of commercial applications in biofuel,

\footnotetext{
*Correspondence: berat.haznedaroglu@boun.edu.tr; ekinatil@buffalo.edu

${ }^{1}$ Department of Chemistry, University at Buffalo, The State University of New York (SUNY), Buffalo, NY 14260, USA

${ }^{4}$ Institute of Environmental Sciences, Bogazici University, 34342 Istanbul, Turkey

Full list of author information is available at the end of the article
}

nutraceutical, pharmaceutical, and environmental sectors [1-3]. High efficiency in photosynthesis, rapid growth in minimized land, and the capability to utilize recycled inputs such as carbon dioxide, nitrogen, and phosphorus from waste streams contribute to sustainability metrics and offer economic feasibility for large-scale commercialization $[4,5]$. One of the key aspects of biotechnological exploitation in microalgae focuses on lipids [6, 7]. In particular, green microalgae species accumulating lipids 
as energy storage molecules under stress conditions have gathered varied interests for potential applications $[5,8$, 9]. Key examples include essential fatty acids (FAs) for nutraceuticals $[10,11]$, triacylglycerols (TAGs) for biodiesel applications [6, 12-14], and carotenoids for nutraceuticals and food additives [15-17].

From a cellular point of view, lipids are not only building blocks for value-added biochemicals but also structural components of cellular membranes and plastids [18-20]. Though not comprehensively explored in algae, lipids and their metabolites also take part in signaling pathways [20-22]. Consequently, understanding lipid trafficking and compartmentalization in microalgae represents an opportunity for improved understanding of cellular functionality and improved biomass yields towards final product optimization.

From a metabolic point view, a majority of green microalgae, generally referred to as oleaginous species, accumulate more lipids under nutrient stress conditions [23-25]. However, the lack of a macronutrient adversely affects cellular homeostasis and, thus, cellular growth stagnates. This is a major topic of interest for algal scientists and researchers investigating optimal conditions leading to higher lipid yields and sustained cellular growth profiles.

Studies in model species such as Chlamydomonas reinhardtii, Chlorella sp., and Nannochloropsis sp. [26-28] have identified key mechanisms of lipid metabolism in green microalgae. Meanwhile, new sets of questions emerged with Nannochloropsis which differs in lipid metabolism among other microalgae species [27, 29]. This was also evident in other species as the regulation of multi-sub-unit enzyme complexes, such as the acetylCoA carboxylase and alcohol dehydrogenase, with roles in fatty acid metabolism differ in response to varying stress conditions [30, 31]. Trafficking of free FAs between chloroplast, cytosol, and endoplasmic reticulum also shows dissimilarity, resulting in an uneven accumulation of lipid bodies that mainly consist of TAGs, important products for biodiesel production, among microalgae species [27, 32-34].

To resolve lipid regulation and metabolic fractionation of lipids in microalgae, high-resolution mass spectrometry (HR-MS) lipidomic studies are required. HR-MS is advantageous in providing information at the molecular level in addition to being utilized in a high-throughput manner to study the composition of complex extracts. In this study, we utilized a liquid chromatography-quadrupole time-of-flight mass spectrometry (LC-QToF MS) based approach developed by our group to analyze differences in lipid composition in the non-model microalga species Ettlia oleoabundans, formerly known as Neochloris oleoabundans [35]. The reason for choosing $E$. oleoabundans as the target species is its native propensity for lipid accumulation up to $35-54 \%$ of its dry cell weight (DCW) without any genetic modification [36, 37]. Due to high quality and quantity of neutral lipids [38], E. oleoabundans is considered as an oleaginous species and a viable candidate for the commercial exploration towards production of biofuels and other value-added products $[39,40]$.

Here, we investigated the effects of nitrogen and phosphorus deplete conditions ( $\mathrm{N}-$ and $\mathrm{P}-$ ) compared to replete conditions $(\mathrm{N}+$ and $\mathrm{P}+)$ in a time-series manner, covering early and late exponential and stationary phases. Combining a targeted and untargeted metabolomics approach with temporal variation in cellular growth, we were able to gain detailed and comprehensive information on the biochemical changes that take place in E. oleoabundans over time and under nutrient stress. This level of detailed characterization both at the temporal and metabolite coverage level in this organism, to the best of our knowledge, has not been carried out before.

\section{Methods \\ Materials}

Liquid chromatography - mass spectrometry (LC-MS) grade methanol and high-performance liquid chromatography (HPLC) grade isopropanol were purchased from Millipore Sigma (Billerica, MA). Formic acid, ammonium formate, and ammonium hydroxide were purchased from Sigma-Aldrich (St. Louis, MO). HPLC grade chloroform was purchased from Honeywell Burdick \& Jackson (Muskegon, MI). Glass beads $(0.5 \mathrm{~mm})$ were purchased from Bertin Technologies (Montigny, France). Stock solutions of lipid standards, purchased from Avanti (Alabaster, $\mathrm{AL}$ ), were prepared in chloroform and kept at $-80{ }^{\circ} \mathrm{C}$ until analysis. The columns for LC-MS analysis were purchased from Phenomenex (Torrance, CA) and included a C5 Luna column $(50 \times 4.6 \mathrm{~mm}, 5 \mu \mathrm{m})$ and a C18 Gemini column $(50 \times 4.6 \mathrm{~mm}, 5 \mu \mathrm{m})$ for electrospray ionization positive (ESI+) and negative (ESI-) modes. Nanopure water was used for all analyses (resistivity 18.2 M $\Omega$-cm at $\left.25^{\circ} \mathrm{C}\right)$.

\section{Experimental methods \\ Microalgal strain, media, and cultivation conditions}

Ettlia oleoabundans (strain UTEX1185 from the culture collection of University of Texas at Austin) was grown under nitrogen replete $(\mathrm{N}+)\left(\left[\mathrm{NO}_{3}{ }^{-}\right]=7.14 \mathrm{mM}\right)$, nitrogen deplete $(\mathrm{N}-)\left(\left[\mathrm{NO}_{3}{ }^{-}\right]=0.714 \mathrm{mM}\right)$, phosphorus replete $(\mathrm{P}+)\left(\left[\mathrm{PO}_{4}{ }^{3-}\right]=1.808 \mathrm{mM}\right)$, and phosphorus deplete $(\mathrm{P}-)\left(\left[\mathrm{PO}_{4}{ }^{3-}\right]=0.064 \mathrm{mM}\right)$ conditions. Microalga was grown in modified Bold $3 \mathrm{~N}$ growth medium in $500 \mathrm{~mL}$ photobioreactors (PBRs) for 11 days. Five biological replicates were used for each condition. PBRs were 
used to grow algae at $25 \pm 2{ }^{\circ} \mathrm{C}$, under a light:dark cycle of 14:10 h using fluorescent light (32W Ecolux, General Electric, Fairfield, CT) and there was a $110 \mu$ mol-photon/ $\mathrm{m}^{2} / \mathrm{s}$ photosynthetic photon flux density. A flow rate of $200 \mathrm{~mL} / \mathrm{min}$ using a mass flow controller (Cole-Parmer Instrument Company, IL) was used to aerate the PBRs continuously using sterile, activated carbon-filtered air without additional $\mathrm{CO}_{2}$.

\section{Determination of optical density, nitrate, and phosphate concentrations in the media, dry biomass, and lipid composition}

The optical density and nitrate and phosphate concentrations in the media were analyzed each day, while dry biomass and lipid composition were analyzed at time points of interest, i.e., days $2,4,7$, and 10 , corresponding to early exponential, late exponential, early stationary, and late stationary phases, respectively. The optical density was measured at $730 \mathrm{~nm}$ using a microwell-plate reader (SpectraMax i3, Molecular Devices, CA). The total nitrate and phosphate concentrations in culture media were determined daily by a Microplate Nitrate Kit (NECi, Lake Linden, MI) and a Phosphate Colorimetric Assay Kit (BioVision, Milpitas, CA). The dry biomass was analyzed by filtering $10 \mathrm{~mL}$ of the microalga growth suspension using vacuum filtration and drying at $105^{\circ} \mathrm{C}$ for $2 \mathrm{~h}$. The dry biomass values are reported in Additional file 1 : Table S1.

The lipid composition was calculated by extracting the lipid content of cells by taking $45 \mathrm{~mL}$ of each culture, centrifuging at $2934 \times g$, and resuspending the pellets in $1 \mathrm{~mL}$ of methanol. The suspension was homogenized by bead-milling in a vial containing 0.1 and $0.5 \mathrm{~mm}$ diameter glass beads. The suspension was then transferred to a new glass vial. Next, $5 \mathrm{~mL}$ of 2-ethoxyethanol was mixed with the methanol solution and the vial shaken $(200 \mathrm{rpm})$ at $60{ }^{\circ} \mathrm{C}$ for $30 \mathrm{~min}$. Solid residues were removed by passage through $1.2 \mu \mathrm{m}$ glass microfiber filters. Later, the solvent was evaporated, and the extract was weighed and resuspended in $3 \mathrm{~mL}$ of 2:2:1:1 hexane:toluene:acetone:methanol mixture to remove extracted proteins and other non-lipid compounds. Finally, the solution was decanted, and individual vials were dried at $60{ }^{\circ} \mathrm{C}$ and re-weighed. The percent lipid composition was calculated as (precipitated lipid weight/ total dry weight) * 100 .

\section{Metabolite extraction and biomass normalization}

The hydrophobic metabolites were extracted following a procedure described in detail previously [35]. Briefly, pellets were resuspended in methanol spiked with C18:1 fatty acid- $d 9$ (henceforth referred to as oleic acid- $d 9$ ) and disrupted by bead-milling. The resulting solution was mixed with chloroform and water (resulting in a mixture of 2:1:1 chloroform:methanol:water) for three serial biphasic extractions. The chloroform layers from three extractions were combined. During the extraction, the samples were spiked with TAG (19:0/19:0/19:0) to serve as an additional surrogate to calculate the extraction efficiencies. The combined chloroform layers were then rotary evaporated and resuspended in chloroform. The volume of chloroform used to resuspend these samples varied based on the differences in biomass, such that each sample had approximately $9.20 \mathrm{mg}$ of biomass $/ \mathrm{mL}$.

\section{Extraction efficiency calculations}

The extraction efficiencies of oleic acid- $d 9$ and TAG (19:0/19:0/19:0) were calculated using matrix-matched calibration curves. The abundances of the internal standards in each sample were then used to calculate concentrations based on the matrix-matched calibration curve. The percent recovery was calculated as [measured concentration of surrogate in sample spiked before the extraction $(\mu \mathrm{M}) /$ theoretical concentration $(\mu \mathrm{M})]$ " 100 .

\section{LC-QToF-MS-based data acquisition}

The normalized extracts were analyzed using an Agilent 1260 HPLC unit (equipped with a degasser, binary pump, autosampler, temperature gauge, and isopump) that was coupled to an Agilent 6530 Quadrupole Time-of-Flight (QToF) mass spectrometer (MS) with a Dual Jet Stream electrospray ionization (ESI) source. Two independent profiling experiments with three biological replicates $(n=3)$ from 16 growth conditions $(\mathrm{N}-, \mathrm{N}+, \mathrm{P}-$, and $\mathrm{P}+$ conditions each sampled individually on days 2,4 , 7, and 10) were analyzed both in ESI+ and ESI- ionization modes. The samples were run in a randomized order to prevent any run-dependent biases. Mobile phase A was composed of 95:5 water/methanol mixture and mobile phase B was made of 60:35:5 isopropanol/methanol/water, supplemented with either $0.1 \%$ ammonium hydroxide for ESI- or $0.1 \%$ formic acid and ammonium formate for ESI+. The starting flow rate was $0.1 \mathrm{~mL} /$ min of $100 \% \mathrm{~A}$, which was then increased to $0.5 \mathrm{~mL} / \mathrm{min}$ after $5 \mathrm{~min}$; the mobile phase composition was changed gradually from $100 \%$ A to $100 \%$ B from 5 to $45 \mathrm{~min}$, and was maintained at $100 \% \mathrm{~B}$ for $10 \mathrm{~min}$. The mobile phase was then equilibrated back to $100 \% \mathrm{~A}$ and kept there for $8 \mathrm{~min}$ at $0.5 \mathrm{~mL} / \mathrm{min}$. The total run length was $63 \mathrm{~min}$. For analysis under ESI+, a C5 column was used for chromatographic separation to allow the analysis of highly hydrophobic biodiesel precursor lipids, TAGs, while a C18 column was used for analysis under ESI-. The data acquisition was carried out in extended dynamic range mode and $m / z$ 's between 250 and 1700 were analyzed. 


\section{Untargeted analysis and LC-MS/MS identification}

The resulting raw data were imported into MassHunter Profinder (version B.06.00, Agilent Technologies) for peak alignment and extraction of features. The recursive feature extraction was used to extract and align molecular features in all samples. This was intended for the deconvolution of chromatograms and alignment of molecular features across all sample data files according to mass and retention time. The parameters for the molecular feature extraction included: a peak height of $\geq 300$ ion counts and possible ions including $[\mathrm{M}+\mathrm{H}]^{+}$, $[\mathrm{M}+\mathrm{Na}]^{+},\left[\mathrm{M}+\mathrm{NH}_{4}\right]^{+}$, and $[\mathrm{M}-\mathrm{H}]^{-}$. The alignment also involved isotope grouping restrictions including: a peak spacing tolerance of $0.0025 \mathrm{~m} / z$ and $7.0 \mathrm{ppm}$ and a maximum charge state of 2 . It was also required that there were at least two or more ions for a single molecular feature. In addition, for binning and alignment purposes, a tolerance of $0.3 \mathrm{~min}$ for a retention time window was set along with a mass window of $20 \mathrm{ppm}$. Some of the postprocessing filters included: an absolute height filter of $\geq 3000$ ion counts and the requirement for the molecular feature to be present in at least two out of three replicates in one experimental group. There was also a restriction set for the extracted ion chromatograms of an expected retention time range of $\pm 1.5 \mathrm{~min}$, along with an acceptable data variation (across samples) of $5.6 \mathrm{ppm}$ for the $\mathrm{m} / \mathrm{z}_{\text {observed }}$ and $0.2 \mathrm{~min}$ for the retention time for each molecular feature.

The resulting aligned features were analyzed in Mass Profiler Professional (MPP, version B12.6.1 Agilent Technologies) for statistically significant differentiations between sampling conditions. Briefly, in MPP, the features were first filtered by frequency ( $>66 \%$ or two out of three replicates for at least one sample group). Next, for statistical significance, analysis of variance (ANOVA) at 95\% confidence level with a post hoc Tukey honest significant difference test and Benjamini-Hochberg false discovery rate (FDR) correction was performed. This process was carried out for two independent profiling experiments and the resulting feature lists were compared and the common features were kept. These $m / z$ 's were then manually inspected for mass accuracy and abundance in Qualitative Analysis (version B.06.00 Agilent Technologies), and reintegrated if necessary. The retention time shifts observed between the first and the last sample injected were around $0.5 \mathrm{~min}$. This list was then analyzed using a more stringent filtering and statistical criteria (a frequency of $100 \%$ and a $p$ value of $<0.01$ ). The resulting $m / z$ 's were searched in METLIN [41] and Lipid Maps [42] databases to determine one or more possible candidate metabolites based on accurate mass and adduct. Known lipid standards (or lipids belonging to the same lipid families) were purchased for the candidate lipids. The liquid chromatography-tandem mass spectrometry (LC-MS/ MS) data were collected similar to the descriptions provided in "LC-QToF-MS-based data acquisition" section, except fragments were observed at collision energies of $15,35,55$, and $75 \mathrm{eV}$. The tandem mass spectrometry (MS/MS) fragmentation patterns of the $m / z$ of interest and similar standards were compared. Searches based on MS/MS fragments provided in METLIN were also used in addition to the fragmentation patterns of known standards.

\section{Targeted analysis of endogenous lipids}

Targeted analysis of $m / z$ 's from major lipid families was carried out by extracting the $\mathrm{m} / z$ 's of the most abundant adduct for each lipid family.

\section{Results and discussion}

Growth curves, lipid composition, and nutrient concentrations in the media

Ettlia oleoabundans cells were grown under four different nutrient conditions $(\mathrm{N}-, \mathrm{N}+, \mathrm{P}-$, and $\mathrm{P}+)$ and sampled at days 2, 4, 7, and 10, representing early and late exponential and early and late stationary growth phases, respectively. This matrix allowed insights on temporal changes in the lipid composition during different growth periods and under varying nutrient conditions. Five biological replicates from different PBRs were analyzed for optical density (OD) (Fig. 1a), remaining nitrate $\left(\left[\mathrm{NO}_{3}{ }^{-}\right]\right)$ and phosphate $\left(\left[\mathrm{PO}_{4}{ }^{3-}\right]\right)$ concentrations in the growth media (Fig. 1b, c, respectively), and total lipid composition (Fig. 1d).

Based on the growth curves monitored daily, early exponential (day 2), late exponential (day 4), early stationary (day 7), and late stationary (day 10) growth phases were confirmed (Fig. 1a). Total nitrate and phosphate in growth media were internalized completely by day 2 , confirming the initiation of nutrient-depleted conditions in $\mathrm{N}-$ and $\mathrm{P}-$ reactors; meanwhile, residual nitrate and phosphate remaining in $\mathrm{N}+$ and $\mathrm{P}+$ reactors sustained nutrient replete conditions (Fig. 1b, c). As microalgal cells continued to utilize already internalized nutrients, biomass growth continued until early stationary phase at day 7 (Fig. 1a).

We measured total lipid contents at respective growth phases to assess the impact of varying nutrient conditions on overall biomass composition (Fig. 1d). Changes in the accumulation of total lipids were not statistically significant between $\mathrm{P}-$ vs. $\mathrm{P}+$ except day 2 in $\mathrm{P}+$ reactors. However, we observed significant increases over time in total lipid contents under $\mathrm{N}-$ conditions compared to $\mathrm{N}+$ (Fig. 1d). In general, more lipids accumulated over time, though $\mathrm{P}-$ did not result in significant increases in lipid content of E. oleoabundans. In contrast, $\mathrm{P}+$ 

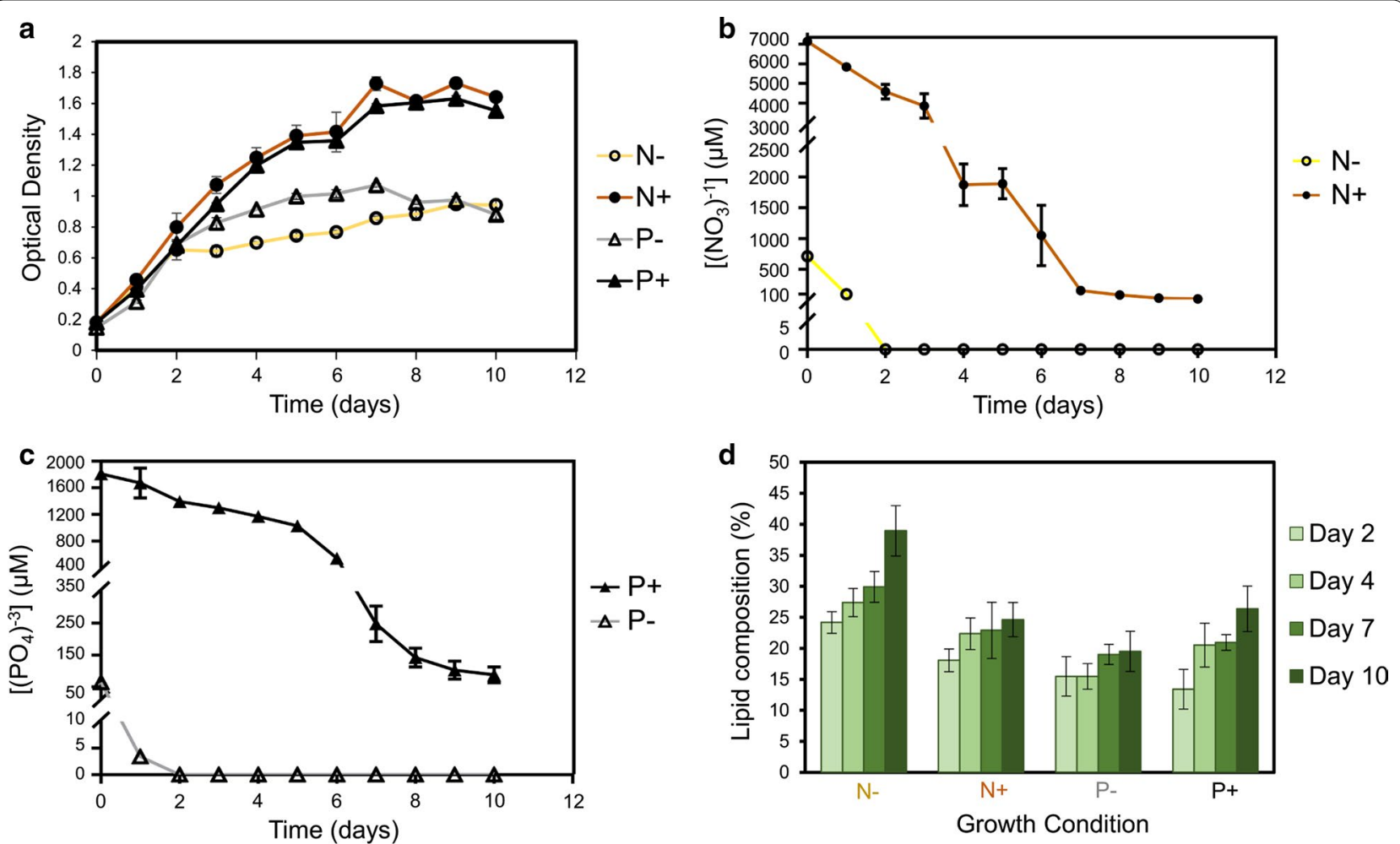

Fig. 1 Growth rate, nitrate and phosphate concentrations in the media, and lipid composition. a Growth rate measured by optical density (at $730 \mathrm{~nm})$. b Nitrate concentrations $(\mu \mathrm{M})$ in media over time. c Phosphate concentrations $(\mu \mathrm{M})$ in media over time. d Lipid composition ( $[$ lipid biomass/total biomass] * 100) of the microalga cells at different time points

reactors showed slightly higher levels of lipids. This was contrary to increased levels of lipid content observed in Chlorella sp. exposed to phosphorus limitation [43]. Xin et al. also observed decreased lipid content with respect to increased phosphorus concentrations in Scenedesmus sp. LX1 [44]. However, there are also reported studies of phosphorus limitation causing decreased lipid content in Nannochloris atomus and Tetraselmis sp. [45]. Meanwhile, N- had a more profound effect similar to the observations of most other green microalgae species accumulating more lipids under $\mathrm{N}-$ (Fig. 1d) [7, 46, 47]. A comparison of lipid accumulation in different green microalgae species exposed to similar nutrient variations is provided in Additional file 1: Table S2. Comparing the changes in different lipid classes, we observe a few major differences between $E$. oleoabundans and other green microalgae species such as $C$. reinhardtii, Chlorella sp., and Nannochloropsis sp. First, monogalactosyldiacylglycerols (MGDGs), which are one of the most abundant plant lipids in green microalgae [48], are depleted under $\mathrm{N}-$ in C. reinhardtii, Chlorella sp., and Nannochloropsis sp. However, these lipids are present at very low levels in E. oleoabundans in the current growth conditions (i.e., only C34:4- and C34:6-MGDGs are detected).
Monogalactosylmonoacylglycerols (MGMGs) and phosphatidic acids (PAs), on the other hand, show significant depletions under $\mathrm{N}-$ in E. oleoabundans, while the previous studies in other species have not reported any changes in their respective levels [27, 28, 49]. In addition, under $\mathrm{N}-$, E. oleoabundans resulted in depletion of phosphatidylglycerols (PGs) and FAs, while in Chlorella sp. PGs [27] and in Nannochloropsis sp. FAs were accumulated [49]. We note that the data on changes in lipid composition under $\mathrm{P}-$ are limited compared to $\mathrm{N}-$, and while we did not observe significant changes in total lipid levels in E. oleoabundans under P-, in Chlorella sp., there was an increase in lipid content [43].

\section{Biomass calculations, extraction, and normalization of metabolites and extraction efficiencies}

Prior to LC-MS analysis, dried biomass values of all samples were calculated for $10 \mathrm{~mL}$ aliquots of algal culture, and extracted microalga were normalized to $9.20 \mathrm{mg}$ of biomass $/ \mathrm{mL}$ in chloroform (Additional file 1: Table $\mathrm{S} 1$, the normalized value was calculated based on the measured biomass provided in "Determination of optical density, nitrate and phosphate concentrations in the media, dry biomass, and lipid composition" section). The 
results of the measurement showed a range of biomass from $0.214 \mathrm{mg} / \mathrm{mL}$ (in one of the biological replicates for $\mathrm{N}-2$ condition) up to $1.478 \mathrm{mg} / \mathrm{mL}$ (in one of the biological replicates for $\mathrm{P}+7$ condition). These measured biomasses and calculated normalization values are shown in Additional file 1: Table S1.

To ensure that lipids were extracted similarly within all experimental conditions and reliable downstream analyses were conducted, we investigated the extraction efficiencies of two internal standards spiked into the samples during different stages of metabolite extraction at the most extreme conditions, i.e., nutrient-deplete on day 2 and -replete on day 10 (Additional file 1: Figure S1A, B). We found that the extraction efficiencies of TAG (19:0/19:0/19:0) were close to $100 \%$ and showed no significant difference (Additional file 1: Figure S1B). On the other hand, we found modest differences in the extraction efficiency of oleic acid- $d 9$ (62-99\%, Additional file 1: Figure $\mathrm{S} 1 \mathrm{~A}$ ), which we attributed to the fact that oleic acid- $d 9$ was spiked at a very early point during extraction and, thus, was slightly affected by sample handling. Overall, the high recovery values for these standards in different experimental conditions showed that the extraction protocol was effective and reproducible for the metabolomics analyses to be performed.

\section{Untargeted metabolomics of the hydrophobic metabolites} The samples grown under varying nitrate $(\mathrm{N}-$ vs. $\mathrm{N}+$ ) and phosphate $(\mathrm{P}-$ vs. $\mathrm{P}+)$ conditions were analyzed separately. Two independent profiling experiments with three biological replicates $(n=3$, highlighted in gray in Additional file 1: Table S1) from all 16 sampling conditions were performed to identify reproducible changes in the hydrophobic metabolite composition (sampling conditions are listed in Additional file 1: Figure S1C). The data analysis for the initial untargeted profiling was performed following a procedure we describe in detail elsewhere ([35], Additional file 1: Figure S2). We focused on $\mathrm{m} / \mathrm{z}$ 's that were present in $100 \%$ of the biological replicates in at least one experimental condition and showed reproducible significant changes $(p<0.01$, see "Experimental methods", "Untargeted analysis and LC-MS/MS identification" section for details, Additional file 1: Figure S2). These analyses resulted in 86 and $39 \mathrm{~m} / z^{\prime}$ s that showed a significant differentiation under varying nitrate and phosphate conditions over time, respectively (Fig. 2, Additional file 1: Figure S2 and Table S3). The greater number of $m / z$ 's identified under $\mathrm{N}-$ vs. P- was consistent with the more profound differences in overall lipid contents under these conditions (Fig. 1d).

We were also interested in pairwise comparisons between experimental conditions and calculated $p$ values for both nutrient variations and different growth phases
(Fig. 2a-d). Figure 2a, b shows the $p$ values for the comparison of the same growth phase (i.e. days 2, 4, 7 and 10) under $\mathrm{N}-$ vs. $\mathrm{N}+$ (Fig. 2a) and $\mathrm{P}-$ vs. $\mathrm{P}+$ (Fig. 2b), respectively. When different nutrient conditions at the same growth period were compared to each other, there was increased significance as the time period increased from day 2 to day 10 for both nitrate and phosphate variations (Fig. 2a, b, respectively), which suggested at the biochemical level that differences in metabolite composition increased from the early exponential to late stationary growth period in E. oleoabundans. This observation was in line with the differences in lipid composition in Fig. 1d which showed that the lipid composition had the greatest difference, especially for nitrate variation at day 10. Comparison of the two different growth periods under the same nutrient condition showed that the differences between day 2 vs. day 10 and day 2 vs. day 7 were the most significant under nitrate variation, while the difference between day 2 vs. day 10 was the most significant for phosphate variation (Fig. 2c, d, respectively).

For the untargeted hydrophobic metabolite sample set, we carried out MS/MS experiments to identify the $\mathrm{m} / z$ 's showing significant differentiation and annotated 70 successfully ( 55 out of 86 under nitrogen variation and 15 out of 36 under phosphorus variation, Fig. 3a, b and Additional file 1: Table S3). The relative abundances of the annotated $m / z$ 's are presented in Fig. 3a, b for nitrate and phosphate conditions, respectively. A majority of these $\mathrm{m} / z^{\prime}$ 's $(\sim 31 \%)$ were identified as chlorophylls and their derivatives (shown in gray in Fig. 3a, b, and c) which showed depletion in $\mathrm{N}-7, \mathrm{~N}-10$, and $\mathrm{P}-10$ conditions. In this analysis, $\sim 29 \%$ of the identified $m / z$ 's were simple lipids such as FAs and their common hydroxylated derivatives [50] shown in green in Fig. 3a, b, and c, $20 \%$ were PCs and their derivatives (shown in brown in Fig. 3a, b, and c), 20\% were comprised of other lipid families such as plant lipids (MGMGs and DGDGs), phytosterols, a monoacylglycerol (MAG), a phytosphingolipid, a quinone derivative, and short peptides (shown in yellow in Fig. 3a, b, and c, Additional file 1: Table S3).

\section{Targeted analysis of endogenous lipids}

Untargeted analyses allowed us to obtain information on changes in hydrophobic metabolite composition at a global level in E. oleoabundans under nitrate and phosphate variations over different growth phases. To better understand the biochemical regulations that are coupled to overall lipid accumulation (Fig. 1d) and identify the lipid groups that contribute to this accumulation at a molecular level, we conducted targeted analyses on representative members from major lipid families. In particular, we focused on FAs, MGMGs, MGDGs, DGDGs, SQDGs, PAs, PGs, and phosphatidylinositols (PIs) in 

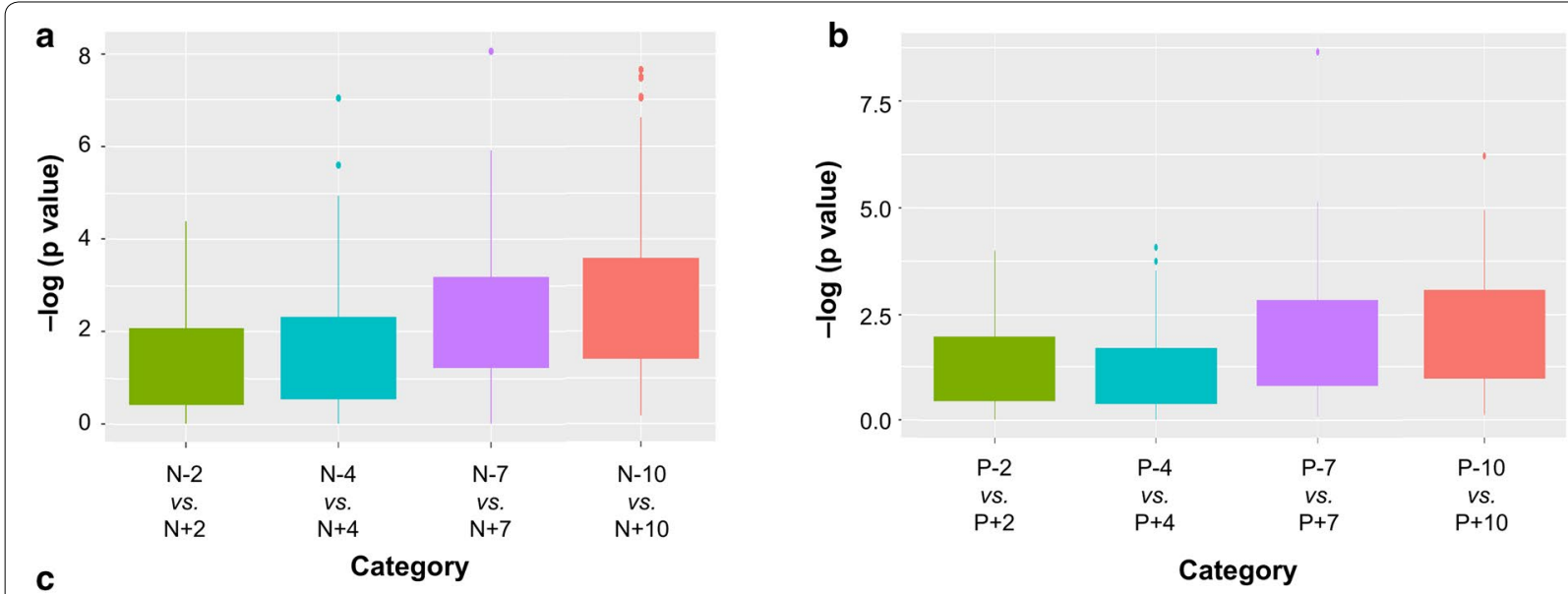

100.0

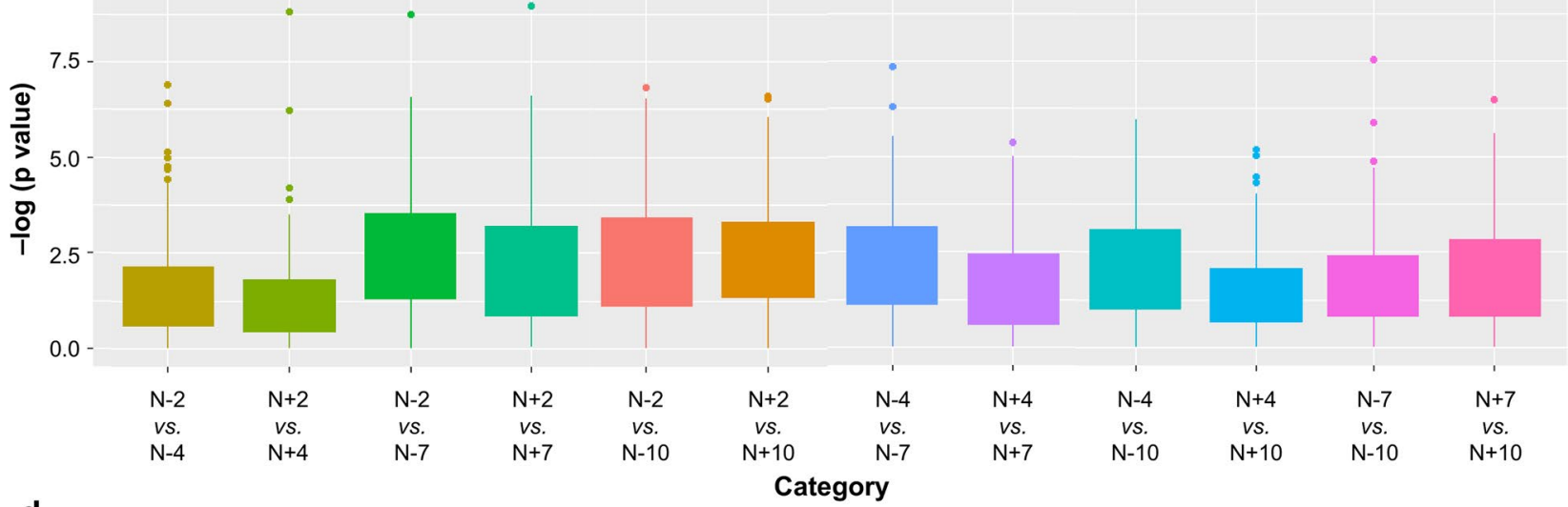

d

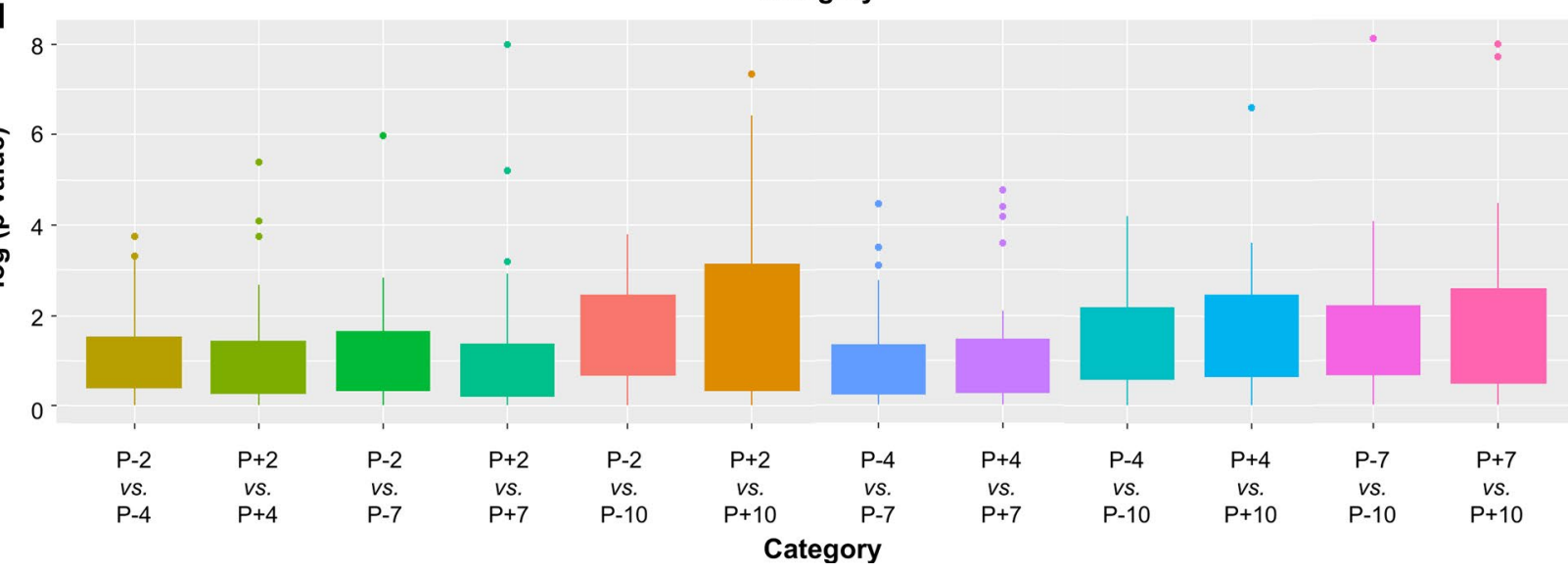

Fig. 2 Nutrient and temporal analysis of significant $m / z^{\prime}$ s detected in E. oleoabundans. $\mathbf{a}-\log (p$ value) of $N-v s$. $N+$ of the same time period. $\mathbf{b}$ $-\log (p$ value $)$ of $\mathrm{P}-\mathrm{vs}$. $\mathrm{P}+$ of the same time period. $\mathbf{c}-\log (p$ value) of varying time points of the same nutrient conditions $(\mathrm{N}-\operatorname{or} \mathrm{N}+) \cdot \mathbf{d}-\log (p$ value) of varying time points of the same nutrient conditions $(\mathrm{P}-$ or $\mathrm{P}+)$

ESI- mode and MAGs, diacylglycerols (DAGs), TAGs, phosphatidylcholines (PCs), and phosphatidylethanolamines (PEs) in ESI+ mode (Additional file 1: Table S3). We targeted lipids from these families with carbon chains ranging from 12 to 24 carbons in length and different unsaturation states. In Fig. 4, we only report lipids that were detected. After confirming their identity by LC-MS/MS, we investigated the changes in abundances under all experimental conditions. Their raw abundances, $m / z$ s, adducts, and observed retention times are provided in Additional file 1: Table S3. 

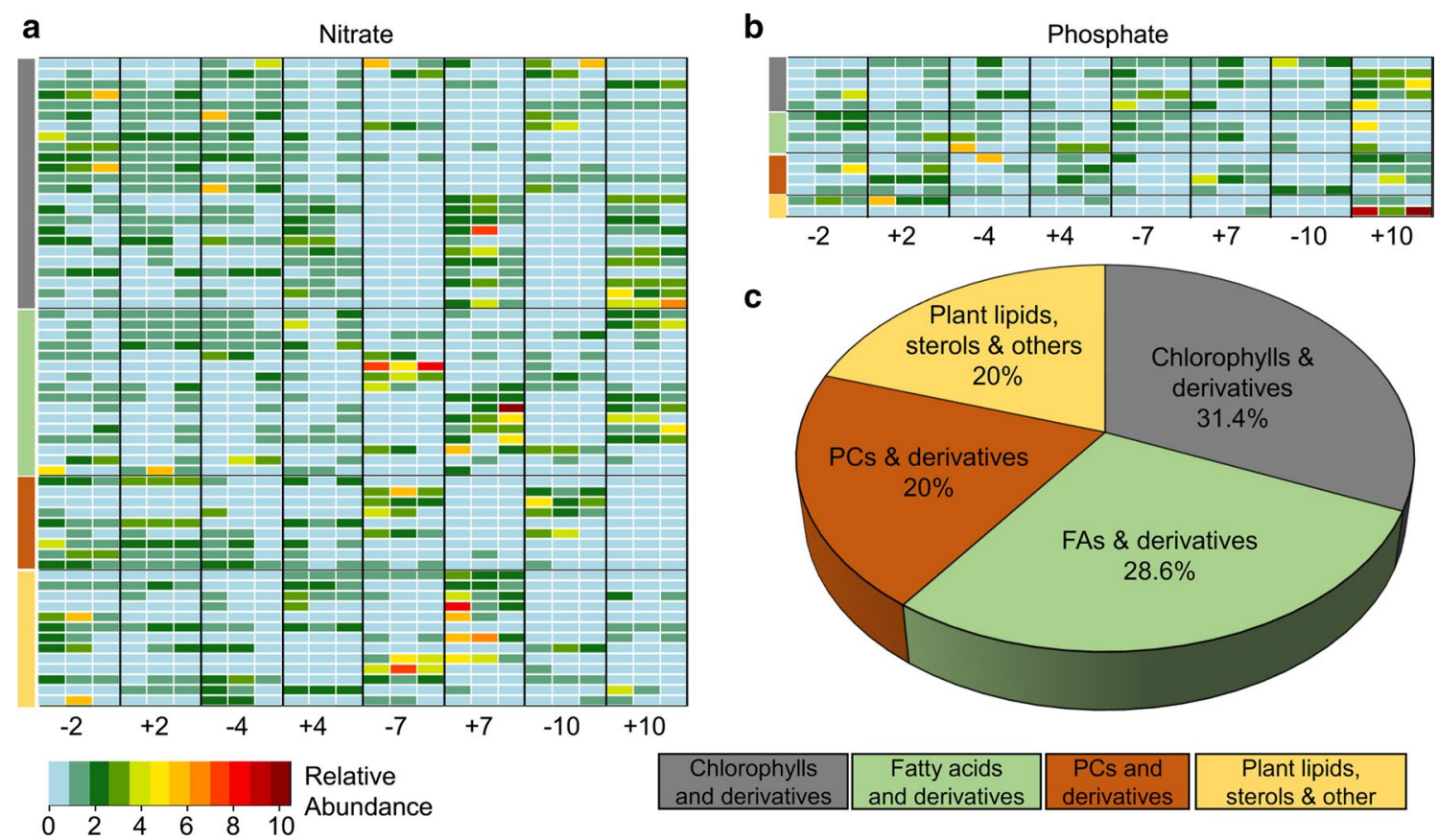

C
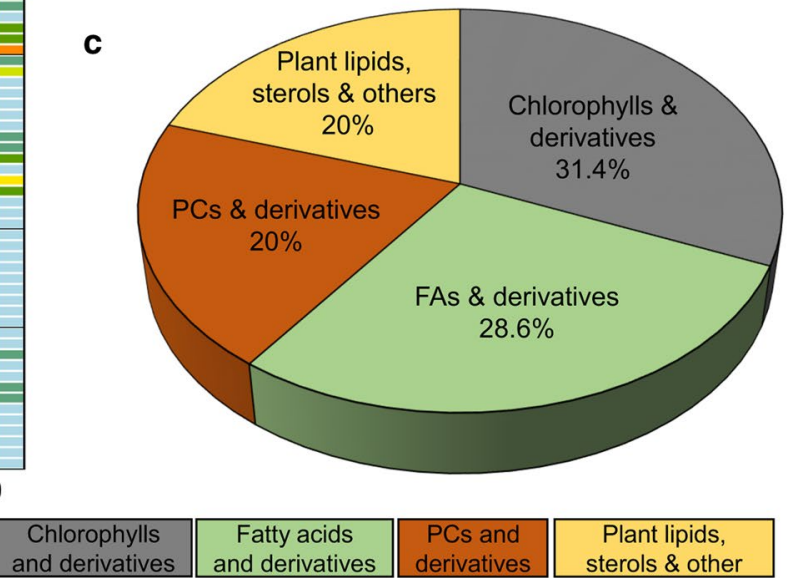

Fig. 3 Hydrophobic metabolites identified under untargeted profiling in E. oleoabundans. $(\mathrm{N}-\mathrm{vs}$. $\mathrm{N}+$ and $\mathrm{P}-\mathrm{vs}$. $\mathrm{P}+$ during different growth periods: day 2, early exponential phase; day 4, late exponential phase; day 7, early stationary phase; and day 10, late stationary phase). a Relative abundances of annotated $\mathrm{m} / \mathrm{z}^{\prime} \mathrm{s}$ that showed significant variations between varying nitrate conditions and time periods. b Relative abundances of annotated $\mathrm{m} / \mathrm{z}^{\prime} \mathrm{s}$ that showed significant variations between varying phosphate conditions and time periods. c Distribution of annotated $\mathrm{m} / \mathrm{z}^{\prime} \mathrm{s}$ presented in $\mathbf{a}$, b based on metabolite family

\section{Changes in fatty acids and glycerolipids}

One of the lipid families that showed alterations at the global level, especially under nitrogen limitation, was FAs and their hydroxylated derivatives. To obtain detailed information regarding the changes in FAs and how these changes might impact other downstream lipids at the molecular level, we targeted different FAs and glycerolipids. We found significant changes in their abundances not only over time but also across nutrient sources (nitrate and phosphate). For instance, specific FAs (i.e., C14:1, C16:2, C16:1, and C18:3) were depleted in $\mathrm{N}$ - over time, whereas they remained similar in $\mathrm{P}-$ and showed some accumulation in $\mathrm{P}+$ (Fig. 4a). Overall, FA accumulation was highest on $\mathrm{N}+7$ and $\mathrm{P}+10$. Deactivation of de novo fatty acid biosynthesis-related enzymes including acyl-CoA carboxylase (ACC) [23, 30, 51, 52], acyl-CoA synthetase (ACS) [52], ATP citrate lyase (ACL), fatty acid synthetase (FAS), and acetyl-CoA C-acetyltransferase (ACAT) [52] under nutrient deprivation over time may be involved in the changes which we observed in FAs. Alternatively, activation of downstream phospholipid or glycerolipid biosynthesis can also cause these depletions.

An intermediate lipid generated during glycerolipid biosynthesis, MAGs, showed no significant changes in $\mathrm{N}-$ or $\mathrm{N}+$ but showed a dynamic regulation under $\mathrm{P}-$.
Their levels first increased at day 4 and day 7 , followed by a depletion at day 10 , which suggests that at the later time points, these lipids were either diverted to different lipids or degraded to replenish free fatty acid pools (Fig. 4a). With respect to DAGs, a significant proportion detected in samples (i.e. C32:1, C32:0, C36:3, C36:2, and C38:5) was accumulated in $\mathrm{N}-7$ and $\mathrm{N}-10$. This was also the case for P-, where DAGs mostly accumulated over time (Fig. 4b). Finally, TAGs, which are synthesized via esterification of DAGs, showed accumulation over time at all analyzed conditions. An interesting observation was $E$. oleoabundans accumulated longer TAGs under $\mathrm{N}-$ and $\mathrm{P}-$ (Fig. 4c), which are usually characteristics of marine microalgae species [53]. Overall, these results support the overall tendency to divert simpler lipids (i.e., FAs, MAGs, and DAGs) for TAG biosynthesis in E. oleoabundans similar to most other oleaginous microalgae species.

\section{Changes in phosphatidylglycerols and other glycerophospholipids}

Phospholipids are important precursors for different modifications including glycosylation. In this study, we targeted different phospholipids, glycerophospholipids, and their lysolipids (i.e., single acylation at the glycerol backbone), and found that there were not only significant 


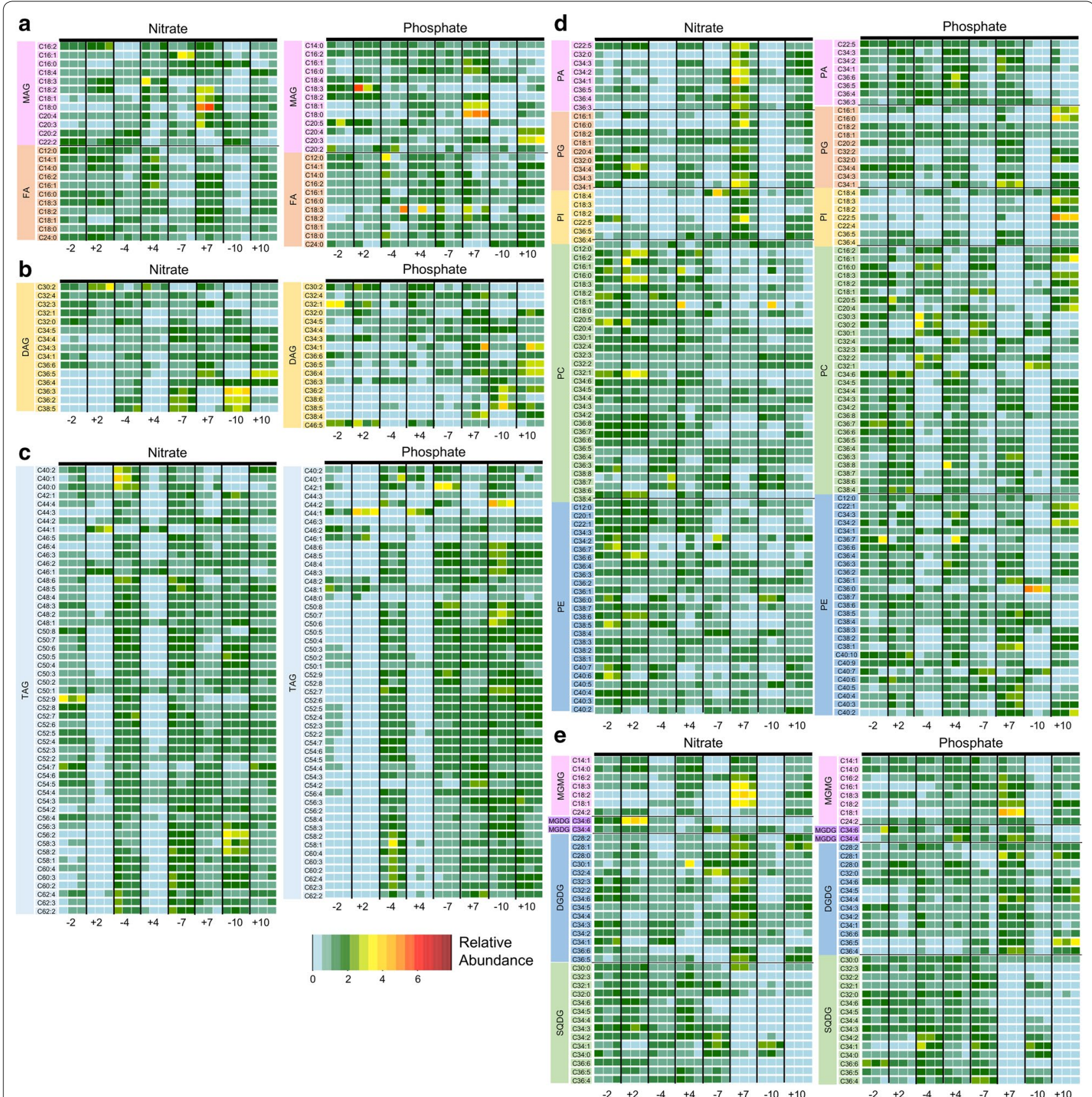

Fig. 4 Targeted analysis results of lipids detected and identified in E. oleoabundans. Relative abundances of a monoacylglycerols (MAGs) and fatty acids (FAs), b diacylglycerols (DAGs), c triacylglycerols (TAGs), d phospholipids e monogalactosylmonoacylglycerols (MGMGs), monogalactosyldiacyl glycerols(MGDGs), digalactosyldiacylglycerols (DGDGs), and sulfoquinovosyldiacylglycerols (SQDGs)

changes between different nutrient conditions $(\mathrm{N}-\mathrm{vs}$. $\mathrm{N}+$ and $\mathrm{P}-$ vs. $\mathrm{P}+$ ) but also between growth phases.

PAs, the simplest glycerophospholipids, detected in E. oleoabundans extracts were depleted in $\mathrm{N}-$ and in $\mathrm{P}-10$ and $\mathrm{P}+10$ (Fig. 4d). PGs and PIs were also depleted in $\mathrm{N}-$ and $\mathrm{P}-$ (Fig. 4d). The depletion of PGs in $\mathrm{N}$ - was surprising, because the previous studies in other green microalgae reported their depletion under $\mathrm{N}+$ $[27,28]$. PCs, another sub-class of glycerophospholipids, were depleted in $\mathrm{N}-$ and $\mathrm{P}-$ over time (i.e., C34:2-34:6, C36:3-36:8, and C38:4-38:8), whereas lysoPCs did not follow a specific trend (Fig. 4d). This was also the case for PEs: some PEs (i.e., C34:2-34:3, C36:1-36:7, C38:138:3, C40:9-40:10, and C40:2-40:4) were depleted, while 
others (i.e., C36:0, C38:4-38:7 and C40:5-40:7) were accumulated in $\mathrm{P}-$ (Fig. $4 \mathrm{~d}$ ).

Based on the changes which we observed in phospholipids and glycerophospholipids, we propose that when the nutrient levels are depleted in E. oleoabundans, there is also a depletion of PAs and PGs in upstream phospholipid biosynthesis. It is also possible that FAs and DAGs were used to "replenish" these glycerophospholipid pools. Since PAs can also functions as signaling molecules [54], its depletion might have negatively affected the synthesis of other lipid families and/or cellular homeostasis. These observations need to be further verified with proteomic and transcriptomic characterizations.

\section{Changes in chlorophylls and PGs under nitrate limitation}

Under nutrient-limited conditions, cellular proliferation rate and overall photosynthetic activity in microalgae decrease $[20,55]$. We observed a gradual depletion of chlorophylls from $\mathrm{N}-2$ to $\mathrm{N}-10$ and their gradual accumulation from $\mathrm{N}+2$ to $\mathrm{N}+10$, which constituted the majority of the changes that we identified based on untargeted analysis (Fig. 3). This could be due to the fact that under $\mathrm{N}-$, microalgal cells were deprived of nitrogen, one of the building blocks of the porphyrin core. We observed a similar trend in PGs (Fig. 4d), which are important structural lipids found in the thylakoid membrane where photosynthetic reactions and electron transport take place [56-59]. For ease of comparison, we summarize these changes in Additional file 1: Figure S3A, B. Based on these observations, it is highly likely that, in addition to the decreased levels of chlorophyll and derivatives under nutrient limitation over time, the depletion of PGs compromises the integrity of thylakoid membranes [20] and, thus, the photosynthetic efficiency in the chloroplasts decreases under these conditions.

\section{Changes in sulfoquinovosyldiacylglycerols and other plant lipids}

The plant lipidome is rich and complex, encompassing many unique plant-specific lipids $[56,58]$. To characterize this diversity, we targeted different plant lipids and studied the changes in their levels under $\mathrm{N}-, \mathrm{N}+, \mathrm{P}-$, and $\mathrm{P}+$ during different growth stages. Particularly, we targeted MGMGs, MGDGs, DGDGs, and SQDGs and found that most of these lipids were differentially regulated between different growth periods and between different nutrient conditions $(\mathrm{N}-$ vs. $\mathrm{N}+$ and $\mathrm{P}-\mathrm{vs} . \mathrm{P}+$ ) in E. oleoabundans.

MGMGs are the simplest plant lipids with one sugar moiety linked to a MAG. These lipids are depleted starting from late exponential (day 4) to late stationary growth (day 10) phase under N-(Fig. 4e). Even though depletion under $\mathrm{P}-$ was observed at late stationary phase, this depletion was preceded by a slight accumulation at early stationary phase (day 7). MGDGs, DAGs with one sugar group at the glycerol backbone, on the other hand, did not show any significant changes between $\mathrm{N}-$ vs. $\mathrm{N}+$, $\mathrm{P}-$ vs. $\mathrm{P}+$, or different growth phases (Fig. 4e). DGDGs, which are MGDGs with an additional sugar group, show a similar trend as MGMGs with a depletion starting from late exponential (day 4) to late stationary (day 10) phase under $\mathrm{N}-$ and depletion from early (day 7 ) to late stationary (day 10) phase in $\mathrm{P}-$ (Fig. 4e).

Next, we analyzed the levels of SQDGs, which are derivatives of MGDGs with sulfonated sugar moieties $[56,60]$. These lipids are found in photosynthetic plants, algae, and other organisms, and are located in the thylakoid membranes [56]. The previous studies have shown their accumulation under $\mathrm{N}+[26-28]$, as well as their degradation under sulfur depletion to repurpose sulfur for the synthesis of new proteins in C. reinhardtii $[61,62]$. Our study showed that SQDGs remain at sustained levels until early stationary phase. After this time point, they show an overall depletion both under nutrient replete and deplete conditions; however, surprisingly, their depletion is more pronounced under $\mathrm{N}+$ and $\mathrm{P}+$ compared to $\mathrm{N}-$ and $\mathrm{P}-$ at late stationary phase (day 10) (Fig. 4e). We inferred from these results that based on the decreased nutrient concentration at day 10 for $\mathrm{N}+$ and $\mathrm{P}+\left(\left[\mathrm{NO}_{3}{ }^{-}\right]=153.7 \mu \mathrm{M}, \mathrm{N}+;\left[\mathrm{PO}_{4}{ }^{3-}\right]=247.7 \mu \mathrm{m}, \mathrm{P}+\right)$, E. oleoabundans might be utilizing SQDGs to provide the necessary building blocks (such as sulfur) for protein synthesis and other components that are necessary to maintain cellular homeostasis.

\section{Changes in saturated fatty acid (SFA), monounsaturated fatty acid (MUFA), and polyunsaturated fatty acid (PUFA) fatty acids (FAs) as compared to phosphatidylglycerols (PGs), digalactosyldiacylglycerols (DGDGs), and sulfoquinovosyldiacylglycerols (SQDGs)}

When comparing the levels of different lipids, FAs are of great interest, since they are the building blocks for the synthesis of downstream lipids. To obtain insight on the changes of FAs and other lipids based on their unsaturation state, we produced a heatmap showing relative abundances of different FAs, PGs, DGDGs, and SQDGs (Additional file 1: Figure S4). We grouped different lipids as saturated fatty acid-(SFA), monounsaturated fatty acid-(MUFA), and polyunsaturated fatty acid-(PUFA) containing lipids. To properly classify lipids as monounsaturated or polyunsaturated lipids, we grouped those with a total of one double bond on the acyl chains as MUFAs and those with a total of three or more double bonds as PUFAs.

MUFAs and PUFAs when grown under $\mathrm{N}-$ decrease in abundance over time as can be seen by the decrease 
in their relative abundance during day 7 and day 10 (early and late exponential phase), while SFAs gradually decrease in abundance over time both under $\mathrm{N}+$ or $\mathrm{N}-$. Comparing the changes in FAs under $\mathrm{N}-$ vs. $\mathrm{N}+$ shows that C14:1, C16:1, and C18:1 FAs are accumulated in $\mathrm{N}+4$ and $\mathrm{N}+7$ and $\mathrm{N}+10$ (late exponential; early and late stationary phases) as compared to the $\mathrm{N}-4, \mathrm{~N}-7$, and $\mathrm{N}-10$ growth conditions. While these trends are apparent under nitrate growth conditions, there seems to be no significant alterations in FAs over time in phosphate growth conditions.

SFA-, MUFA-, and PUFA-PGs decrease in abundance over time when grown under either $\mathrm{N}-$ or $\mathrm{P}-$, while SFA- and MUFA-PGs increase in abundance under $\mathrm{N}+$ and $\mathrm{P}+$ at early and late stationary phases.

The plant lipids which we analyzed show decreased levels under N-. Specifically, SFA- and PUFA-DGDGs when grown under $\mathrm{N}+$ increase in abundance over time, while MUFA- and PUFA-DGDGs when grown under $\mathrm{P}+$ increase in abundance over time. However, PUFADGDGs when grown under $\mathrm{N}-$ decrease in abundance over time. SQDG, on the other hand, exhibits differing trends: MUFA-SQDGs increase over time under $\mathrm{N}-$ and $\mathrm{P}-$, and were depleted under $\mathrm{P}+$. PUFA-SQDGs when grown under all four growth conditions decrease in abundance over time. Overall these trends in PGs, DGDGs, and SQDGs could be attributed to the gradual depletion over time of the SFAs when grown under $\mathrm{N}+$ and $\mathrm{N}-$ and the depletion over time of the MUFAs and PUFAs when grown under $\mathrm{N}-$. However, based on our data, we are limited in explaining how E. oleoabundans utilized FAs for the production of downstream lipids under nutrient limitation. Though the biochemical machinery that controls the incorporation of different FAs to other complex lipids is well defined in plants, this seems to be more complex in green microalgae [48].

\section{Overall analysis of hydrophobic metabolites}

Lipidomics studies carry great importance and have been performed on several microalgae species grown under nutrient replete vs. deplete conditions. A majority of these analyses have only focused on TAG accumulation and how nitrogen limitation affects its synthesis [63]. Under nitrogen deprivation, MGDGs, DGDGs, SQDGs, and PGs were depleted, while TAGs were accumulated in C. reinhardtii [28]. Similarly, in Chlorella sp., MGDGs, DGDGs, SQDGs, PEs, and PCs were depleted, while PGs and TAGs were accumulated under nitrogen deprivation [27]. Another model green microalga, Nannochloropsis sp., when grown under nitrogen deprivation, showed depletions of MGDGs, DGDGs, SQDGs, PCs, PGs, and PIs, the accumulation of TAGs, and no significant trends for PEs [26].
In this study, we carried out a time-series characterization of the effect of different nutrient limitation conditions on lipid composition in a highly adaptable and oleaginous $E$. oleoabundans non-model microalgae. Using a combination of targeted and untargeted lipidomics, we analyzed the levels and detected 377 unique lipids which allowed us to obtain information at the global and metabolic pathway level. In particular, we analyzed the effects of $\mathrm{N}-$ and $\mathrm{P}-$ during early exponential, late exponential, early stationary, and late stationary phases on lipid composition. This is the first comprehensive time-series analysis of $\mathrm{N}-$ vs. $\mathrm{N}+$ and $\mathrm{P}-$ vs. $\mathrm{P}+$ in a non-model green microalga which provided a dynamic picture of the changes in lipid families with respect to cellular growth.

A summary of global changes in lipid composition is provided in Fig. 5. To illustrate the time-dependent changes in a given lipid family under different conditions, each box represents the average relative abundances of all lipids that we targeted in a given family. Based on this, the optimal growth conditions for each lipid family are as follows: $\mathrm{N}-2$ for PEs, $\mathrm{N}+2$ for PCs and MGDGs, P -4 for SQDGs, $\mathrm{N}+7$ for PAs, PGs, and FAs, $\mathrm{P}+10$ for PIs, either $\mathrm{N}+7$ or $\mathrm{P}+7$ for DAGs, DGDGs, and MGDGs, and $\mathrm{N}-7$ or $\mathrm{N}-10$ or $\mathrm{P}+7$ or $\mathrm{P}+10$ for TAGs. We show that a strong accumulation of TAGs, critical precursors for biodiesel production, and depletion of chlorophylls occur under deprivation as cells reach late stationary phase (Fig. 5).

Multiple enzymes might contribute to the changes in glycerolipids. These include glycerol-3-phosphate O-acyltransferase (GPAT) [52, 64], 1-acyl-sn-glycerol-3-phosphate O-acyltransferase (AGPAT) [51, 52], lysophosphatidic acid acyltransferase (LPAAT) [64], phosphatidic acid phosphatase (PP or PAP) [51, 52, 64], and diacylglycerol acyltransferase (DGAT-1 and DGAT2) $[23,30,51,52,64]$ and phospholipid: diacylglycerol acyltransferase (PDAT) $[23,52]$, which have been correlated to changes in the MAG, DAG, and TAG abundance trends that are seen when grown under $\mathrm{N}-$ and $\mathrm{P}-$ as compared to $\mathrm{N}+$ and $\mathrm{P}+$. We envision that the activation of DGAT-1 and DGAT-2 plays important roles in the strong accumulation of TAG accumulation may stem from an over expression of DGAT-1 or 2.

In addition, phospholipids showed regulation based on acyl chain composition, except for PGs which gradually decreased over time in nutrient-depleted conditions (Fig. 5). This depletion of PGs most likely affected the chloroplast and thylakoid membrane structure and the production of chlorophylls, which in turn decreased overall photosynthetic efficiency of $E$. oleoabundans as supported by the growth profiles (Fig. 1a). Finally, we observed a non-conventional regulation of SQDGs, 


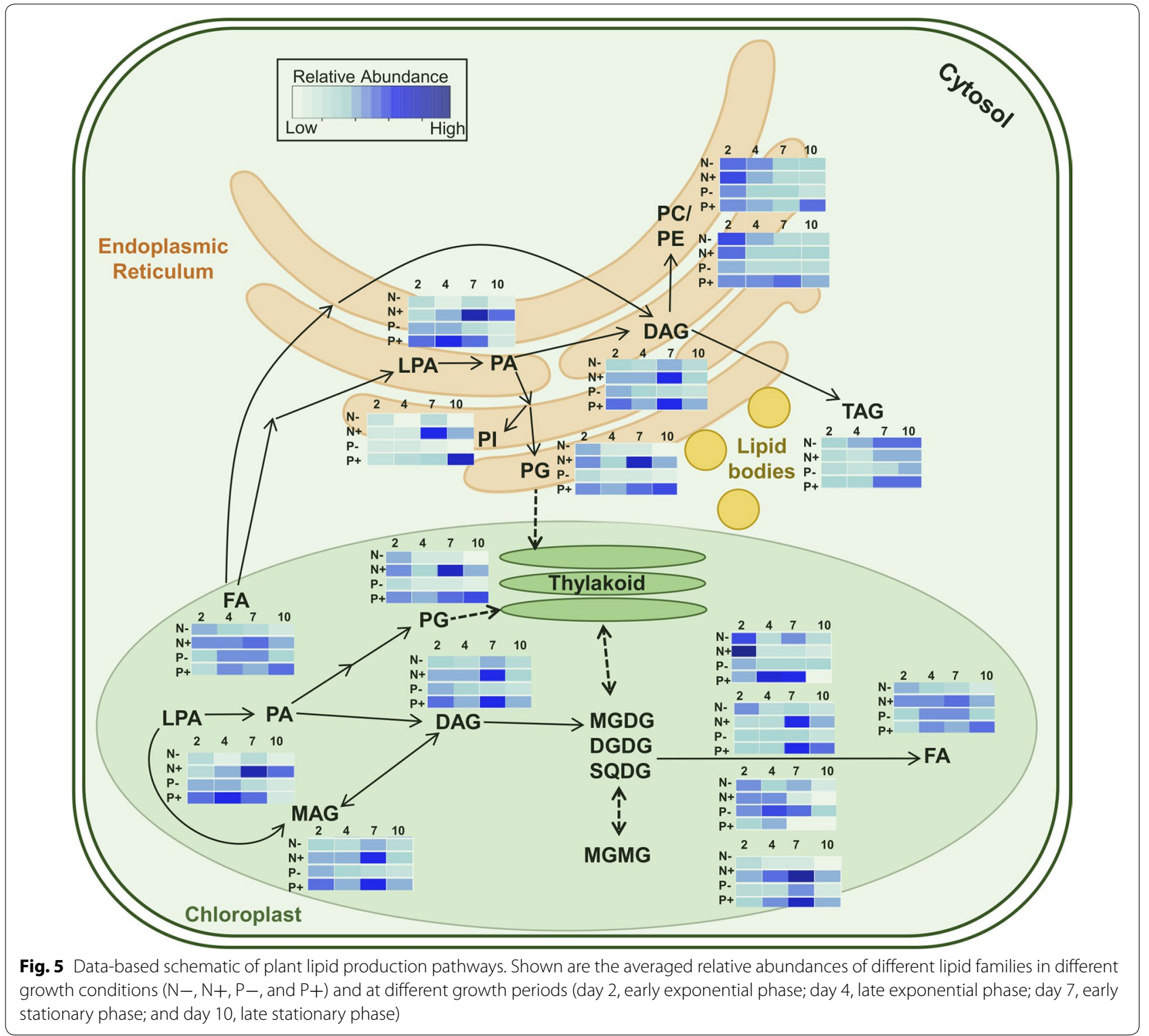

which were depleted under $\mathrm{N}+$ and $\mathrm{P}+$ during the stationary phase (Fig. 5). We reason that this could be due to a switch in metabolism to utilize sulfur for the synthesis of essential biomolecules for cellular survival under nutrient stress. Intriguing questions remain to be answered on the biochemical machinery that regulates these changes. For instance, UDP-sulfoquinovose synthase (SQD1) [23], sulfoquinovosyldiacylglycerol synthases, (SQD2 or SQD3) [23], monogalactosyldiacylglycerol synthases (MGD1 or MGDGS) [23, 64], and digalactosyldiacylglycerol synthases (DGD1 or DGDGS) [23, 64] could be involved in the changes in plant lipids which we observed under $\mathrm{N}-$ or $\mathrm{P}-$. Understanding the role of individual enzymes and biosynthetic pathways will be the key to elucidate important metabolic transitions under nutrient deprivation.

\section{Conclusions}

In this work, we conducted the first time-series characterization of the changes in the lipidome under nutrient deprivation in a non-model oleaginous microalga. Integrating targeted and untargeted analyses, we captured the temporal regulation and overall accumulation of TAGs from early exponential to late stationary phases, which is advantageous for biodiesel production in E. oleoabundans, as well as novel regulations including ones in structural and plant-specific lipids. These results clearly show that there is a complex regulation of lipids under 
nutrient variation with respect to cellular growth in the microalga species. Future studies that include information on more species and experimental conditions elucidating this complexity will provide invaluable insights into the optimized production of value-added products including biodiesel in green microalgae.

\section{Additional file}

Additional file 1. Three additional figures and two additional tables.

\begin{abstract}
Abbreviations
TAGs: triacylglycerols; DGDGs: digalactosyldiacylglycerols; SQDGs: sulfoquinovosyldiacylglycerols; PGs: phosphatidylglycerols; FAs: fatty acids; HR-MS: high-resolution mass spectrometry; LC-QToF-MS: liquid chromatography-quadrupole time-of-flight mass spectrometry; DCW: dry cell weight; $\mathrm{N}$-: nitrogen deplete; $\mathrm{P}-$ : phosphorus deplete; $\mathrm{N}+$ : nitrogen replete; $\mathrm{P}+$ : phosphorus replete; LC-MS: liquid chromatography-mass spectrometry; HPLC: high-performance liquid chromatography; ESI: electrospray ionization; $\mathrm{ESI}+$ : positive mode; $\mathrm{ESI}-$ : negative mode; $\mathrm{NO}_{3}{ }^{-}$: nitrate; $\mathrm{PO}_{4}{ }^{3-}$ : phosphate; PBRs: photobioreactors; MPP: Mass Profiler Professional; ANOVA: analysis of variance; FDR: false discovery rate; LC-MS/MS: liquid chromatography-tandem mass spectrometry; MS/MS: tandem mass spectrometry; MGMGs: monogalactosylmonoacylglycerols; MGDGs: monogalactosyldiacylglycerols; PAs: phosphatidic acids; PIs: phosphatidylinositols; MAGs: monoacylglycerols; DAGs: diacylglycerols; PCs: phosphatidylcholines; PEs: phosphatidylethanolamines; SFAs: saturated fatty acids; MUFAs: monounsaturated fatty acids; PUFAs: polyunsaturated fatty acids; ACC: acyl-CoA carboxylase; ACS: acyl-CoA synthetase; ACL: ATP citrate lyase; FAS: fatty acid synthetase; ACAT: acetyl-CoA C-acetyltransferase; GPAT: glycerol-3-phosphate O-acyltransferase; AGPAT: 1-acyl-sn-glycerol-3-phosphate O-acyltransferase; LPAAT: lysophosphatidic acid acyltransferase; PP: phosphatidate phosphatase; DGAT-1 and DGAT-2: diacylglycerol acyltransferase; PDAT: phospholipid: diacylglycerol acyltransferase; SQD1: UDP-sulfoquinovose synthase; SQD2 or 3: sulfoquinovosyldiacylglycerol synthase; MGD1 or MGDGS: monogalactosyldiacylglycerol synthase; DGD1 or DGDGS: digalactosyldiacylglycerol synthase.
\end{abstract}

\section{Authors' contributions}

The experiments were designed by GEA-G, BZH, and BAP. The experiments were conducted by EKM, MG, and EC, and EKM carried out the MS and MS/MS analyses. The manuscript was written by EKM, EC, BZH, BAP, and GEA-G. The study was directed by BZH and GEA-G. All authors read and approved the final manuscript.

\begin{abstract}
Author details
${ }^{1}$ Department of Chemistry, University at Buffalo, The State University of New York (SUNY), Buffalo, NY 14260, USA. ${ }^{2}$ Department of Civil, Structural and Environmental Engineering, University at Buffalo, The State University of New York (SUNY), Buffalo, NY 14260, USA. ${ }^{3}$ Department of Chemical and Biological Engineering, Koc University, 34450 Istanbul, Turkey. ${ }^{4}$ Institute of Environmental Sciences, Bogazici University, 34342 Istanbul, Turkey. ${ }^{5}$ Department of Chemical and Biological Engineering, University at Buffalo, The State University of New York (SUNY), Buffalo, NY 14260, USA.
\end{abstract}

\section{Acknowledgements}

We would like to thank Virginia del Solar for the assistance in lipid identifications.

\section{Competing interests}

The authors declare that they have no competing interests.

\section{Availability of data and materials}

The data sets used and/or analyzed during the current study are available from the corresponding author on reasonable request.
Consent for publishing

Not applicable.

Ethics approval and consent to participate

Not applicable.

\section{Funding}

This research was supported by the National Science Foundation (Award No: CBET-1438172) and Boğaziçi University BAP Research Fund (Award No: 14Y00SUP10). B.Z.H is a Newton Advanced Fellow and supported by Royal Society of UK (Award No: NA140481).

\section{Publisher's Note}

Springer Nature remains neutral with regard to jurisdictional claims in published maps and institutional affiliations.

Received: 7 August 2017 Accepted: 17 January 2018

Published online: 06 February 2018

\section{References}

1. Bux F, Chisti Y. Algae biotechnology: products and processes. Berlin: Springer; 2016.

2. Richmond A, Hu Q. Handbook of microalgal culture: applied phycology and biotechnology. New York: Wiley; 2013.

3. Rosales-Mendoza S. Perspectives for the algae-made biopharmaceuticals field. In: Algae-based biopharmaceuticals. Berlin: Springer; 2016. p. 143-63.

4. Singh B, Bauddh K, Bux F. Algae and environmental sustainability. Algae Environ Sustain Ser Dev Appl Phycol. 2015;7:155-64.

5. Torres CM, Ríos SD, Torras C, Salvadó J, Mateo-Sanz JM, Jiménez L. Microalgae-based biodiesel: a multicriteria analysis of the production process using realistic scenarios. Bioresour Technol. 2013;147:7-16.

6. Hu Q, Sommerfeld M, Jarvis E, Ghirardi M, Posewitz M, Seibert M, Darzins A. Microalgal triacylglycerols as feedstocks for biofuel production: perspectives and advances. Plant J. 2008;54(4):621-39.

7. Rodolfi L, Chini Zittelli G, Bassi N, Padovani G, Biondi N, Bonini G, Tredici MR. Microalgae for oil: strain selection, induction of lipid synthesis and outdoor mass cultivation in a low-cost photobioreactor. Biotechnol Bioeng. 2009;102(1):100-12.

8. Mujtaba G, Choi W, Lee C-G, Lee K. Lipid production by Chlorella vulgaris after a shift from nutrient-rich to nitrogen starvation conditions. Bioresour Technol. 2012;123:279-83.

9. Negi S, Barry AN, Friedland N, Sudasinghe N, Subramanian S, Pieris S, Holguin FO, Dungan B, Schaub T, Sayre R. Impact of nitrogen limitation on biomass, photosynthesis, and lipid accumulation in Chlorella sorokiniana. J Appl Phycol. 2016;28(2):803-12.

10. Adarme-Vega TC, Lim DK, Timmins M, Vernen F, Li Y, Schenk PM. Microalgal biofactories: a promising approach towards sustainable omega-3 fatty acid production. Microb Cell Fact. 2012;11(1):96.

11. Qin J. Lipid-containing secondary metabolites from algae. In: Handbook of hydrocarbon and lipid microbiology. Berlin: Springer; 2010. p. 3021-30.

12. Brennan L, Owende P. Biofuels from microalgae-a review of technologies for production, processing, and extractions of biofuels and coproducts. Renew Sustain Energy Rev. 2010;14(2):557-77.

13. Liu B, Benning C. Lipid metabolism in microalgae distinguishes itself. Curr Opin Biotechnol. 2013;24(2):300-9.

14. Pienkos PT, Darzins A. The promise and challenges of microalgal-derived biofuels. Biofuel Bioprod Biorefin. 2009;3(4):431-40.

15. Delgado-Vargas F, Jiménez A, Paredes-López O. Natural pigments: carotenoids, anthocyanins, and betalains - characteristics, biosynthesis, processing, and stability. Crit Rev Food Sci. 2000;40(3):173-289.

16. Dufossé L, Galaup P, Yaron A, Arad SM, Blanc P, Murthy KNC, Ravishankar GA. Microorganisms and microalgae as sources of pigments for food use: a scientific oddity or an industrial reality? Trends Food Sci Technol. 2005;16(9):389-406.

17. Gantar M, Svirčev Z. Microalgae and cyanobacteria: food for thought. J Phycol. 2008;44(2):260-8. 
18. Harwood JL. Membrane lipids in algae. In: Lipids in photosynthesis: structure, function and genetics. Berlin: Springer; 1998. p. 53-64.

19. Hitchcock C, Nichols BW. Plant lipid biochemistry. The biochemistry of fatty acids and acyl lipids with particular reference to higher plants and algae. Dublin: Academic Press, Inc.; 1971.

20. Nakamura Y, Li-Beisson Y. Lipids in plant and algae development. Berlin: Springer; 2016.

21. Guschina IA, Harwood JL. Lipids and lipid metabolism in eukaryotic algae. Prog Lipid Res. 2006;45(2):160-86.

22. Magnin-Robert M, Le Bourse D, Markham J, Dorey S, Clement C, Baillieul F, Dhondt-Cordelier S. Modifications of sphingolipid content affect tolerance to hemibiotrophic and necrotrophic pathogens by modulating plant defense responses in arabidopsis. Plant Physiol. 2015;169(3):2255-74.

23. Boyle NR, Page MD, Liu B, Blaby IK, Casero D, Kropat J, Cokus SJ, Hong-Hermesdorf A, Shaw J, Karpowicz SJ. Three acyltransferases and nitrogen-responsive regulator are implicated in nitrogen starvationinduced triacylglycerol accumulation in Chlamydomonas. J Biol Chem. 2012;287(19):15811-25.

24. Campos H, Boeing WJ, Dungan BN, Schaub T. Cultivating the marine microalga Nannochloropsis salina under various nitrogen sources: effect on biovolume yields, lipid content and composition, and invasive organisms. Biomass Bioenergy. 2014;66:301-7.

25. Griffiths MJ, van Hille RP, Harrison ST. The effect of degree and timing of nitrogen limitation on lipid productivity in Chlorella vulgaris. Appl Microbiol Biotechnol. 2014;98(13):6147-59.

26. Li J, Han D, Wang D, Ning K, Jia J, Wei L, Jing X, Huang S, Chen J, Li Y, et al. Choreography of transcriptomes and lipidomes of Nannochloropsis reveals the mechanisms of oil synthesis in microalgae. Plant Cell. 2014;26(4):1645-65.

27. Martin GJ, Hill DR, Olmstead IL, Bergamin A, Shears MJ, Dias DA, Kentish SE, Scales PJ, Botte CY, Callahan DL. Lipid profile remodeling in response to nitrogen deprivation in the microalgae Chlorella sp. (Trebouxiophyceae) and Nannochloropsis sp. (Eustigmatophyceae). PLoS ONE. 2014;9(8):e103389.

28. Siaut M, Cuine S, Cagnon C, Fessler B, Nguyen M, Carrier P, Beyly A, Beisson F, Triantaphylides C, Li-Beisson Y, Peltier G. Oil accumulation in the model green alga Chlamydomonas reinhardtii: characterization, variability between common laboratory strains and relationship with starch reserves. BMC Biotechnol. 2011:11:7.

29. Vieler A, Brubaker SB, Vick B, Benning C. A lipid droplet protein of Nannochloropsis with functions partially analogous to plant oleosins. Plant Physiol. 2012;158(4):1562-9.

30. Fan J, Cui Y, Wan M, Wang W, Li Y. Lipid accumulation and biosynthesis genes response of the oleaginous Chlorella pyrenoidosa under three nutrition stressors. Biotechnol Biofuels. 2014;7(1):17.

31. Valenzuela J, Mazurie A, Carlson RP, Gerlach R, Cooksey KE, Peyton BM, Fields MW. Potential role of multiple carbon fixation pathways during lipid accumulation in Phaeodactylum tricornutum. Biotechnol Biofuels. 2012;5(1):40.

32. Goold H, Beisson F, Peltier G, Li-Beisson Y. Microalgal lipid droplets: composition, diversity, biogenesis and functions. Plant Cell Rep. 2015;34(4):545-55.

33. Merchant SS, Kropat J, Liu B, Shaw J, Warakanont J. TAG, You're it! Chlamydomonas as a reference organism for understanding algal triacylglycerol accumulation. Curr Opin Biotechnol. 2012;23(3):352-63.

34. Mühlroth $A$, Li K, Røkke G, Winge P, Olsen Y, Hohmann-Marriott MF, Vadstein O, Bones AM. Pathways of lipid metabolism in marine algae, co-expression network, bottlenecks and candidate genes for enhanced production of EPA and DHA in species of Chromista. Mar Drugs. 2013;11(11):4662-97.

35. Matich EK, Butryn DM, Ghafari M, del Solar V, Camgoz E, Pfeifer BA, Aga DS, Haznedaroglu BZ, Atilla-Gokcumen GE. Mass spectrometry-based metabolomics of value-added biochemicals from Ettlia oleoabundans. Algal Res. 2016;19:146-54.

36. Chisti Y. Biodiesel from microalgae. Biotechnol Adv. 2007:25(3):294-306.

37. Pruvost J, Van Vooren G, Cogne G, Legrand J. Investigation of biomass and lipids production with Neochloris oleoabundans in photobioreactor. Bioresour Technol. 2009;100(23):5988-95.
38. Tornabene TG, Holzer G, Lien S, Burris N. Lipid-composition of the nitrogen starved green-alga Neochloris-oleoabundans. Enzyme Microb Technol. 1983;5(6):435-40.

39. Adams C, Godfrey V, Wahlen B, Seefeldt L, Bugbee B. Understanding precision nitrogen stress to optimize the growth and lipid content tradeoff in oleaginous green microalgae. Bioresour Technol. 2013;131:188-94.

40. Gouveia L, Oliveira AC. Microalgae as a raw material for biofuels production. J Ind Microbiol Biotechnol. 2009;36(2):269-74.

41. Zhu ZJ, Schultz AW, Wang J, Johnson CH, Yannone SM, Patti GJ, Siuzdak G. Liquid chromatography quadrupole time-of-flight mass spectrometry characterization of metabolites guided by the METLIN database. Nat Protoc. 2013;8(3):451-60.

42. Fahy E, Sud M, Cotter D, Subramaniam S. LIPID MAPS online tools for lipid research. Nucleic Acids Res. 2007;35(suppl 2):W606-12.

43. Liang K, Zhang Q, Gu M, Cong W. Effect of phosphorus on lipid accumulation in freshwater microalga Chlorella sp. J Appl Phycol. 2013;25(1):311-8.

44. Xin L, Hong-ying H, Ke G, Ying-xue S. Effects of different nitrogen and phosphorus concentrations on the growth, nutrient uptake, and lipid accumulation of a freshwater microalga Scenedesmus sp. Bioresour Technol. 2010;101(14):5494-500.

45. Reitan KI, Rainuzzo JR, Olsen Y. Effect of nutrient limitation on fatty acid and lipid content of marine microalgae. J Phycol. 1994;30(6):972-9.

46. Griffiths MJ, Harrison ST. Lipid productivity as a key characteristic for choosing algal species for biodiesel production. J Appl Phycol. 2009;21(5):493-507.

47. Griffiths MJ, van Hille RP, Harrison ST. Lipid productivity, settling potential and fatty acid profile of 11 microalgal species grown under nitrogen replete and limited conditions. J Appl Phycol. 2012;24(5):989-1001.

48. Li-Beisson Y, Nakamura Y, Harwood J. Lipids: from chemical structures, biosynthesis, and analyses to industrial applications. In: Lipids in plant and algae development. Berlin: Springer; 2016. p. 1-18.

49. Simionato D, Block MA, La Rocca N, Jouhet J, Marechal E, Finazzi G, Morosinotto T. The response of Nannochloropsis gaditana to nitrogen starvation includes de novo biosynthesis of triacylglycerols, a decrease of chloroplast galactolipids, and reorganization of the photosynthetic apparatus. Eukaryot Cell. 2013;12(5):665-76.

50. Kendel M, Barnathan G, Fleurence J, Rabesaotra V, Wielgosz-Collin G. Nonmethylene interrupted and hydroxy fatty acids in polar lipids of the alga Grateloupia turuturu over the four seasons. Lipids. 2013;48(5):535-45.

51. Goh FQY, Jeyakani J, Cazenave-Gassiot A, Tipthara P, Yeo Z, Wenk M, Clarke ND. Core features of triacylglyceride production in Ettlia oleoabundans revealed by lipidomic and gene expression profiling under distinct induction conditions. Algal Res. 2017;26:453-62.

52. Rismani-Yazdi H, Haznedaroglu BZ, Hsin C, Peccia J. Transcriptomic analysis of the oleaginous microalga Neochloris oleoabundans reveals metabolic insights into triacylglyceride accumulation. Biotechnol Biofuels. 2012;5(1):74.

53. Bigogno C, Khozin-Goldberg I, Boussiba S, Vonshak A, Cohen Z. Lipid and fatty acid composition of the green oleaginous alga Parietochloris incisa, the richest plant source of arachidonic acid. Phytochemistry. 2002:60(5):497-503.

54. Goedhart J, Gadella TWJ. Photolysis of caged phosphatidic acid induces flagellar excision in Chlamydomonas. Biochemistry. 2004;43(14):4263-71.

55. Markou G, Vandamme D, Muylaert K. Microalgal and cyanobacterial cultivation: the supply of nutrients. Water Res. 2014;65:186-202.

56. Boudiere L, Michaud M, Petroutsos D, Rebeille F, Falconet D, Bastien O, Roy S, Finazzi G, Rolland N, Jouhet J, et al. Glycerolipids in photosynthesis: composition, synthesis and trafficking. Biochim Biophys Acta. 2014;1837(4):470-80

57. Laczko-Dobos H, Ughy B, Toth SZ, Komenda J, Zsiros O, Domonkos I, Parducz A, Bogos B, Komura M, Itoh S, Gombos Z. Role of phosphatidylglycerol in the function and assembly of photosystem II reaction center, studied in a cdsA-inactivated PAL mutant strain of Synechocystis sp. PCC6803 that lacks phycobilisomes. Biochim Biophys Acta. 2008;1777(9):1184-94.

58. Mizusawa N, Wada H. The role of lipids in photosystem II. Biochim Biophys Acta. 2012;1817(1):194-208.

59. Sato N, Suda K, Tsuzuki M. Responsibility of phosphatidylglycerol for biogenesis of the PSI complex. Biochim Biophys Acta. 2004;1658(3):235-43.

60. Benning C. Biosynthesis and function of the sulfolipid sulfoquinovosyl diacylglycerol. Annu Rev Plant Physiol Plant Mol Biol. 1998;49:53-75. 
61. Sugimoto K, Sato N, Tsuzuki M. Utilization of a chloroplast membrane sulfolipid as a major internal sulfur source for protein synthesis in the early phase of sulfur starvation in Chlamydomonas reinhardtii. FEBS Lett. 2007;581(23):4519-22.

62. Yang D, Song D, Kind T, Ma Y, Hoefkens J, Fiehn O. Lipidomic analysis of Chlamydomonas reinhardtii under nitrogen and sulfur deprivation. PLoS ONE. 2015;10(9):e0137948.

63. Breuer $G$, Lamers PP, Martens DE, Draaisma RB, Wijffels RH. The impact of nitrogen starvation on the dynamics of triacylglycerol accumulation in nine microalgae strains. Bioresour Technol. 2012;124:217-26.
64. Jaeger D, Winkler A, Mussgnug JH, Kalinowski J, Goesmann A, Kruse O. Time-resolved transcriptome analysis and lipid pathway reconstruction of the oleaginous green microalga Monoraphidium neglectum reveal a model for triacylglycerol and lipid hyperaccumulation. Biotechnol Biofuels. 2017:10:197.

\section{Submit your next manuscript to BioMed Central and we will help you at every step:}

- We accept pre-submission inquiries

- Our selector tool helps you to find the most relevant journal

- We provide round the clock customer support

- Convenient online submission

- Thorough peer review

- Inclusion in PubMed and all major indexing services

- Maximum visibility for your research

Submit your manuscript at

www.biomedcentral com/submit 